\title{
Unterschiedliches Risikoprofil bei MVT- und VTE-Patienten
}

Salim S et al. Clinical implications of different risk factor profiles in patients with mesenteric venous thrombosis and systemic venous thromboembolism:

a population-based study. J Thromb Thrombolys 2019; 47: 572-577

Die Mesenterialvenenthrombose (MVT) ist eine seltene und potenziell tödlich verlaufende Erkrankung. Es ist unbekannt, ob das Risikoprofil für die MVT dem für venösen Thromboembolismus (VTE) entspricht. Die schwedische Arbeitsgruppe gingen dieser Frage anhand einer populationsbasierten Studie nach.

In Malmö, Schweden, wurden populationsbasierte Studien zu den beiden venösen thrombotischen Erkrankungen MVT und VTE durchgeführt. Daraus ergab sich die einzigartige Gelegenheit, Unterschiede bei den erworbenen und angeborenen Risikofaktoren beider Patientengruppen zu analysieren. Die MVT-Patienten $(n=120)$ wurden den Patientenakten und dem AuriculA (schwedisches Register für antikoagulativ behandelte Patienten) entnommen. Dabei wurden alle Fälle berücksichtigt, die zwischen Januar 2000 und Dezember 2015 in der Skåne-Universitätsklinik behandelt wurden. Das Follow-up endete am 6. September. Die mittlere Nachverfolgungszeit betrug 6,2 Jahre bei einem Median von 5,4 Jahren (IQR 22,6 Jahre). Die VTE-Gruppe ( $n=1452)$ ent- nahm die Arbeitsgruppe der MATS-Studie (Malmö-Thrombophilie-Studie). In die prospektive populationsbasierte Studie wurden zwischen 1998 und 2008 alle konsekutiven in Malmö behandelten VTE-Patienten unselektiert aufgenommen. Ein Follow-up erfolgte bis zum Tod oder dem Ende der Studie im September 2017. Die mittlere Nachverfolgungszeit betrug 10,2 Jahre bei einem Median von 11,4 Jahren (IQR 6,5-13,7 Jahre).

Die Analyse ergab für die MVT-Gruppe ein anderes Risikoprofil als für die VTE-Gruppe. In 6 Fällen wurde eine tödlich verlaufende MVT bei der Autopsie entdeckt. Alle MVTPatienten wiesen Symptome auf. Sie waren jünger, hatten eine höhere glomeruläre Filtrationsrate, einen niedrigeren Anteil an Rauchern und waren in der jüngeren Vorgeschichte seltener operiert worden. Auch wiesen sie im Vergleich zur VTE-Gruppe häufiger solide Karzinome (19,2\% versus $12,1 \%)$ und intraabdominelle Karzinome auf (16,7\% versus 2,3\%). Im Vergleich zur VTE-Gruppe war die Prävalenz von FaktorV-Mutation Typ Leiden ohne gleichzeitig bestehendes Karzinom in der MVT-Gruppe niedriger (26,6\% versus 38,9\%). Die 30-Ta-
ges-Mortalität war in der MVT-Gruppe mit $10,8 \%$ höher als in der VTE-Gruppe mit $0,5 \%$. Im langfristigen Follow-up ergab die Kaplan-Meier-Analyse jedoch keine Unterschiede bei der Mortalität.

FAZIT

Aus dem unterschiedlichen Risikoprofil beider Gruppen leitet die Arbeitsgruppe 2 Empfehlungen ab: Bei MVTPatienten sollten intraabdominelle Karzinome ausgeschlossen werden. In beiden Gruppen sollte bei Personen ohne Karzinom ein Screening auf Thrombophilie erfolgen, wenn nicht klinisch vorab eine dauerhafte Antikoagulation verordnet wurde. Einschränkend sei die retrospektive Datenerhebung bei der MVT-Gruppe im Vergleich zur prospektiven Datenerhebung bei der VTE-Gruppe zu berücksichtigen.

Dr. Gabriele Dobler, Berlin 